Ilchmann, Achim ; Owens, David H. :

Exponential stabilization using nondifferential gain adaptation

Zuerst erschienen in:

IMA Journal of Mathematical Control and Information 7 (1991), S. 339-349 


\title{
Exponential Stabilization using Nondifferential Gain Adaptation
}

\author{
AChim IlchmanN \\ Institut für Angewandte Mathematik, Universität Hamburg, \\ D.2000 Hamburg 13, Germany \\ David H. Owens \\ University of Exeter, School of Engineering, \\ North Park Road, Exeter EX4 $4 Q F$, UK
}

[Received 12 January 1990]

\begin{abstract}
A new type of adaptive high-gain stabilizer for classes of linear time-invariant state-space systems is presented. The classes cover multi-input multi-output systems where the state dimension is not known. Only standard assumptions of minimum phase and known (respectively unknown) sign are required. The controller is of nondifferential form and ensures exponential decay of the trajectory. In the case where the sign of the high-frequency gain is not known, a new type of switching function is introduced that guarantees switching convergence independent of gain adaptation.
\end{abstract}

\section{Notation}

$\begin{array}{ll}\mathbb{R}_{+} & =\{s \in \mathbb{R}: s>0\} \\ \mathbb{C}_{+} & =\{s \in \mathbb{C}: \operatorname{Re} s>0\} \\ \mathbb{C}_{-} & \{s \in \mathbb{C}: \operatorname{Re} s<0\} \\ \sigma(A) & =\left\{\lambda_{1}(A), \ldots, \lambda_{n}(A)\right\}=\text { spectrum of } A \in \mathbb{R}{ }^{n \times n} \\ \mathrm{~L}_{r}(0, t) & =\text { set of all } r \text {-integrable functions } f:(0, t) \rightarrow \mathbb{R}^{n} \\ \mathrm{~L}_{x}(0, t) & =\text { set of all bounded functions } f:(0, t) \rightarrow \mathbb{R}^{n} \\ \mathrm{C}\left([-h, 0], \mathbb{R}^{n}\right)= & \text { set of all continuous functions } f:[-h, 0] \rightarrow \mathbb{R}^{n}, \\ & n \text { being defined by the context. }\end{array}$

\section{Introduction}

OVER THE past few years, the area of adaptive high-gain stabilization has been concerned with the derivation of a number of control schemes of simple time-varying adaptive output feedback (see, to name but a few, Morse (1983), Nussbaum (1983), Byrnes \& Willems (1984), Mårtensson (1986), Ilchmann et al. (1987), Prätzel-Wolters et al. (1989)). In all cases, the controllers guarantee asymptotic stabilization of the output $y(t)$ via adaptive gain selection obtained by solving a differential equation (e.g. $\dot{k}=y^{2}$ ). In this paper, two issues are addressed: the derivation of gain adaptation rules of nondifferential form that are capable of ensuring exponential decay of the system input and state with a computable decay rate for the output magnitude. In order to achieve this, a 
so-called 'maximum controller'

$$
k(t)=\sup _{0 \leqslant s \leqslant t} \mathrm{e}^{\omega(s) s}|y(s)|,
$$

with suitable choice of $\omega(\cdot)$, is introduced together with a switching decision function of an entirely new form.

We consider a class of time-invariant linear systems of the form

$$
\left.\begin{array}{l}
\dot{x}(t)=A x(t)+B u(t), \\
y(t)=C x(t),
\end{array}\right\}
$$

where $A \in \mathbb{R}^{n \times n}$, and $B, C^{\top} \in \mathbb{R}^{n \times m}$ are unknown, but have to satisfy the high-frequency gain condition

$$
\operatorname{det} C B \neq 0
$$

together with the minimum-phase condition

$$
\operatorname{det}\left[\begin{array}{cc}
s I-A & -B \\
C & 0
\end{array}\right] \neq 0 \quad \text { for all } s \in \overline{\mathbb{C}}_{+} .
$$

The state dimension $n$ of the system need not be known.

For multi-input multi-output systems, we will consider the known-sign case when (1.3) is strengthened to

$$
\sigma(C B) \subset \mathbb{C}_{+} .
$$

(Note: the case of $\sigma(C B) \subset \mathbb{C}_{-}$is a trivial extension of this case obtained by input scaling.) For single-input single-output systems, we also treat the unknown-sign case $C B \neq 0$.

In Section 2, we consider adaptive stabilization of multi-input multi-output systems of the form (1.2) which satisfy the minimum-phase condition (1.4) and the spectral condition (1.5) on the high-frequency gain. This section also introduces the maximum controller and illustrates the natural structure of the control for the purposes of exponential stabilization.

In Section 3, this idea is extended to the general case of unknown sign of $C B$ for single-input single-output systems. In this context, the original concept of a Nussbaum gain is replaced by a new switching decision function that guarantees the convergence of the switching characteristics in finite time independent of the choice of gain adaptation.

\section{Multi-input multi-output systems with known sign at high frequencies}

In this section, we consider the class $\Sigma$ of systems of the form (1.2) which satisfy the minimum-phase condition (1.4) and the known-sign condition

$$
\sigma(C B) \subset \mathbb{C}_{+} .
$$

Before we state the main result, some investigations are undertaken into the closed-loop system

$$
\dot{x}(t)=[A-k(t) B C] x(t)
$$


which arises if the feedback law

$$
u(t)=-k(t) y(t)
$$

is applied to a system of the form (1.2).

Lemma 2.1 Suppose ( $A, B, C)$ satisfies the conditions (1.2)-(1.4), and the linear output feedback

$$
u(t)=-k(t) y(t)
$$

where $k(\cdot):\left[0, t^{\prime}\right) \rightarrow \mathbb{R}$ is a piecewise continuous function, with $t^{\prime} \leqslant \infty$, is applied to the system. Then the closed-loop system

$$
\dot{x}(t)=[A-k(t) B C] x(t)
$$

can be converted, by a suitable coordinate transformation, into the form

$$
\left.\begin{array}{l}
\dot{y}(t)=\left[A_{1}-k(t) C B\right] y(t)+A_{2} \eta(t), \\
\dot{\eta}(t)=A_{3} y(t)+A_{4} \eta(t),
\end{array}\right\}
$$

where $A_{1} \in \mathbb{R}^{m \times m}, A_{2}, A_{3}^{\top} \in \mathbb{R}^{m \times(n-m)}, A_{4} \in \mathbb{R}^{(n-m) \times(n-m)}$, and

$$
\sigma\left(A_{4}\right) \subset \mathbb{C}_{-} \text {. }
$$

Moreover, if $k(t)$ is monotonically increasing to infinity as $t \rightarrow t^{\prime}\left(t^{\prime} \leqslant \infty\right)$ and (1.5) is satisfied, then

$$
\|\phi(t, s)\| \leqslant M \mathrm{e}^{\left(-\varepsilon+\delta_{0}\right)(t-s)} \text { for all } t^{\prime}>t \geqslant s \geqslant t_{0},
$$

where $\phi(\cdot, \cdot)$ denotes the transition matrix of $(2.4), M>0$ is some constant independent of $t_{0}, \varepsilon>0$ so that $\operatorname{Re} \lambda_{i}\left(A_{4}\right)<-\varepsilon$ for all $i \in \underline{n}$, and $\delta_{0}=\delta\left(t_{0}\right)>0$ is monotonically decreasing to zero as $k\left(t_{0}\right)$ tends to infinity.

Proof. See Owens et al. (1987).

Lemma 2.1 enables the following result to be proved for an exponentially stabilizing 'maximum control scheme'.

Proposition 2.2 Suppose the system $(A, B, C)$ belongs to $\Sigma$ and $h>0$. Consider the 'maximum control scheme'

$$
\begin{aligned}
& k(t)=\max _{0 \leqslant s \leqslant t} \mathrm{e}^{\omega(s) s}\|y(s)\|+k_{0} \quad\left(k_{0} \geqslant 0\right), \\
& \omega(t)=\left\{\begin{array}{cl}
1 & \text { for } t \in[0, h], \\
\frac{1}{1+k(t-h)} & \text { for } t>h,
\end{array}\right. \\
& u(t)=-k(t) y(t) .
\end{aligned}
$$

Then the closed-loop system

$$
\dot{x}(t)=[A-k(t) B C] x(t), \quad x(0)=x_{0}
$$


satisfies gain convergence in the sense that

$$
\lim _{t \rightarrow \infty} k(t)=k_{\infty}<\infty
$$

and for some $M, \lambda>0$ we have the exponential bound

$$
\|x(t)\| \leqslant M \mathrm{e}^{-\lambda t} \text { for all } t \geqslant 0 .
$$

Proof. The differential equation (2.9) is of the nonlinear delay type. Using the notation

(2.9) reads

$$
x_{t}(\cdot):=x(t+\cdot), \quad \omega_{t}(\cdot):=\omega(t+\cdot)
$$

$$
\dot{x}(t)=f\left(t, x_{t}\right), \quad x(0)=x_{0}
$$

where

$$
f\left(t, x_{t}\right)= \begin{cases}A x_{t}(0) & \text { for } t<0, \\ {\left[A-\left(\max _{s \in[-t, 0]} \mathrm{e}^{t+s}\left\|C x_{t}(s)\right\|+k_{0}\right) B C\right] x_{t}(0)} & \text { for } t \in[0, h], \\ {\left[A-\left(\max _{s \in[-t, 0]} \mathrm{e}^{\omega_{t}(s)}\left\|C x_{t}(s)\right\|+k_{0}\right) B C\right] x_{t}(0)} & \text { for } t>h .\end{cases}
$$

Note that $f\left(\cdot, x_{t}\right)$ may be discontinuous at $t=h$. However, without restriction of generality, we may assume that $k_{0}=0$. This can be done since $f\left(\cdot, x_{t}\right)$ satisfies the Carathéodory condition (see Hale, 1977: p. 55) and the existence and uniqueness of (2.12) goes through as for the case of continuous $f\left(\bullet, x_{t}\right)$.

We proceed in several steps and prove the following statements.

(i) If there exists a solution of $(2.12)$ on $\left(-\infty, t^{\prime}\right)$, with $t^{\prime} \leqslant \infty$, then $k(\cdot) \epsilon$ $\mathrm{L}_{\infty}\left(0, t^{\prime}\right)$.

(ii) System (2.12) does not have a finite escape time.

(iii) A solution of (2.12) exists on the whole of $\mathbb{R}$ and is unique.

(iv) There exist $M, \omega>0$ such that the solution $x(\cdot)$ of (2.9) satisfies

$$
\|x(t)\| \leqslant M \mathrm{e}^{-\omega t} \text { for all } t \geqslant 0
$$

and $(2.10)$ is fulfilled.

(i) Suppose there exists a solution $x(\cdot)$ of $(2.12)$ on $\left(-\infty, t^{\prime}\right)$ and $k(\cdot) \notin \mathrm{L}_{\infty}\left(0, t^{\prime}\right)$. Then Lemma 2.1 implies that

$$
|| x(t) \mid \leqslant K \mathrm{e}^{-\lambda t} \text { for all } t \in\left[0, t^{\prime}\right)
$$

for some $K, \lambda>0$. Since $k(t)$ is monotonically increasing in $t$, it follows that $\lim _{t \rightarrow t^{\prime}} \omega(t)=0$. Thus, (2.13) applied to

$$
k(t)=\max _{0 \leq s \leq t} \mathrm{e}^{\omega(s) s}\|y(s)\|+k_{0}
$$

yields $k(\cdot) \in \mathrm{L}_{\infty}\left(0, t^{\prime}\right)$. Hence (i) is proved by a contradiction argument. 
(ii) Suppose there exists some $t^{\prime}>0$ such that

$$
\limsup _{t \rightarrow t^{\prime}}\|x(t)\|=\infty \text {. }
$$

Since, by (i), $k(\cdot) \in \mathrm{L}_{\infty}\left(0, t^{\prime}\right)$, it follows that (2.9) satisfies a global Lipschitz condition on $\left(0, t^{\prime}\right)$. This contradicts (2.14), and hence (ii) is proved.

(iii) Consider the continuous map

$$
f: \Omega=(-\infty, h) \times \mathrm{C}\left([-h, 0], \mathbb{R}^{n}\right) \rightarrow \mathbb{R}^{n}
$$

given by (2.12). Function $f$ is called locally Lipschitz in the second argument if $f$ is Lipschitz in the second argument in each compact set in $\Omega$. Function $f$ is in fact locally Lipschitz as a sum, product, and composition of locally Lipschitz maps. Therefore, the result in Hale (1977: p. 42) can be applied and the existence and uniqueness of the solution $x(t)$ of $(2.12)$ through the initial value $\left(0,\left.x(\cdot)\right|_{|-h, 0|}\right)$ follows for $t \in(-\infty, \varepsilon)$, for some $\varepsilon>0$. Now (ii) implies that the solution can be continued on $(-\infty, h]$. The same arguments yield existence and uniqueness on $(-\infty, 2 h]$. By repeating these arguments inductively, (iii) follows.

(iv) It follows from (i)-(iii) that (2.10) holds true. Therefore $\lim _{t \rightarrow \infty} \omega(t)=$ $\omega_{\infty}>0$ and we obtain

$$
\mathrm{e}^{\omega_{x} t}\|y(t)\| \leqslant \max _{s \in[0, t]} \mathrm{e}^{\omega(s) s}\|y(s)\| \leqslant\left(k_{x}-k_{0}\right) .
$$

Suppose (2.9) is of the form (2.5). By (2.6), there exist $K, \varepsilon>0$ such that

$$
\| \mathrm{e}^{A_{4} t} \mid \leqslant K \mathrm{e}^{-\varepsilon t} \text { for all } t \geqslant 0 \text {. }
$$

An application of (2.15), (2.16), and variations-of-constants to the second equation in (2.5) yields

$$
\begin{aligned}
\|\eta(t)\| & \leqslant K \mathrm{e}^{-\varepsilon t}\|\eta(0)\|+\int_{0}^{t}\left\|A_{3}\right\| K \mathrm{e}^{-\varepsilon(t-s)}\|y(s)\| \mathrm{d} s \\
& \leqslant \mathrm{e}^{-\varepsilon t} K\left(\|\eta(0)\|+\left\|A_{3}\right\|\left(k_{\infty}-k_{0}\right) \int_{0}^{t} \mathrm{e}^{\left(\varepsilon-\omega_{\infty}\right) s} \mathrm{~d} s\right) .
\end{aligned}
$$

Since $\varepsilon$ in (2.16) can be chosen so that $\varepsilon<\omega_{\infty}$, (2.12) yields exponential decay of $\|\eta(\cdot)\|$. This, together with (2.15), implies (2.11). Therefore the proof is complete.

Remark 2.3. Note that the proof of Proposition 2.2 provides an explicit description of the decay of the output in terms of asymptotically computable quantities. Inequality $(2.15)$ yields

$$
\|y(t)\| \leqslant\left(k_{\infty}-k_{0}\right) \mathrm{e}^{-\omega_{x} t} \text { for all } t \geqslant 0 .
$$

\section{Single-input single-output $n$ th-order systems with unknown sign}

In this section, we consider the class $\hat{\Sigma}$ of single-input single-output systems of the form

$$
\left.\begin{array}{l}
\dot{x}(t)=A x(t)+b u(t) \\
y(t)=c x(t)
\end{array}\right\}
$$


where $A \in \mathbb{R}^{n \times n}$, and $b, c^{\top} \in \mathbb{R}^{n}$ are unknown, but are known to satisfy

$$
c b \neq 0
$$

and the minimum-phase condition

$$
\operatorname{det}\left[\begin{array}{cc}
s I_{n}-A & b \\
c & 0
\end{array}\right] \neq 0 \text { for all } s \neq \overline{\mathbb{C}}_{+}
$$

Note that the sign condition (2.1) is weakened to (3.2). For the class $\hat{\Sigma}$, we follow previous results in developing a switching function in order to generate an adaptive scheme. In contrast to the well-known Nussbaum gain (see Nussbaum, 1983; Willems \& Byrnes, 1984), this switching function is a new type guaranteeing convergence in finite time.

The switching function

$$
S(\cdot):[0, \infty) \rightarrow\{-1,1\}
$$

will be implemented into the feedback law via

$$
u(t)=-S(t) k(t) y(t),
$$

and we consider the closed-loop system

$$
\dot{x}(t)=[A-S(t) k(t) b c] x(t),
$$

where $k(\cdot)$ is the maximum controller introduced in (2.8). We follow the intuitive idea that $S(t)$ is searching for the 'correct' sign of the gain $c b$ and, if it finds it, the sign is kept constant and $k(t)$ will thus converge to a finite limit. Then the exponential decay of $|y(t)|$ is obvious.

We now introduce the switching algorithm. Let $k(\cdot):\left[t_{0}, \infty\right) \rightarrow \mathbb{R}_{+}$be a continuous nondecreasing positive function, and $y(\cdot):\left[t_{0}, \infty\right) \rightarrow \mathbb{R}$ a measurable function with $y\left(t_{0}\right) \neq 0$.

Put, assuming $S(\cdot):\left[t_{0}, t\right) \rightarrow\{-1,1\}$ is known,

$$
\psi(t):=\int_{t_{0}}^{t} S(\tau) k(\tau) y(\tau)^{2} \mathrm{~d} \tau / \int_{t_{0}}^{t} y(\tau)^{2} \mathrm{~d} \tau .
$$

We call $\psi(\cdot)$ a switching decision function and it is related to $S(\cdot)$ via the following algorithm.

Algorithm 3.1

$$
i:=0
$$

$$
S\left(t_{i}\right)=1
$$

(*) $t_{i+1}>t_{i}$ is defined by the property

$$
\begin{aligned}
& \quad S\left(t_{i}\right) \psi(t)<(i+1) k\left(t_{0}\right) \text { for } t \in\left(t_{i}, t_{i+1}\right) \\
& \quad S\left(t_{i}\right) \psi\left(t_{i+1}\right)=(i+1) k\left(t_{0}\right) \\
& S(t):=S\left(t_{i}\right) \text { for } t \in\left[t_{0}, t_{i}\right) \\
& S\left(t_{i+1}\right):=-S\left(t_{i}\right) \\
& i:=i+1 \\
& \text { go to (*). }
\end{aligned}
$$




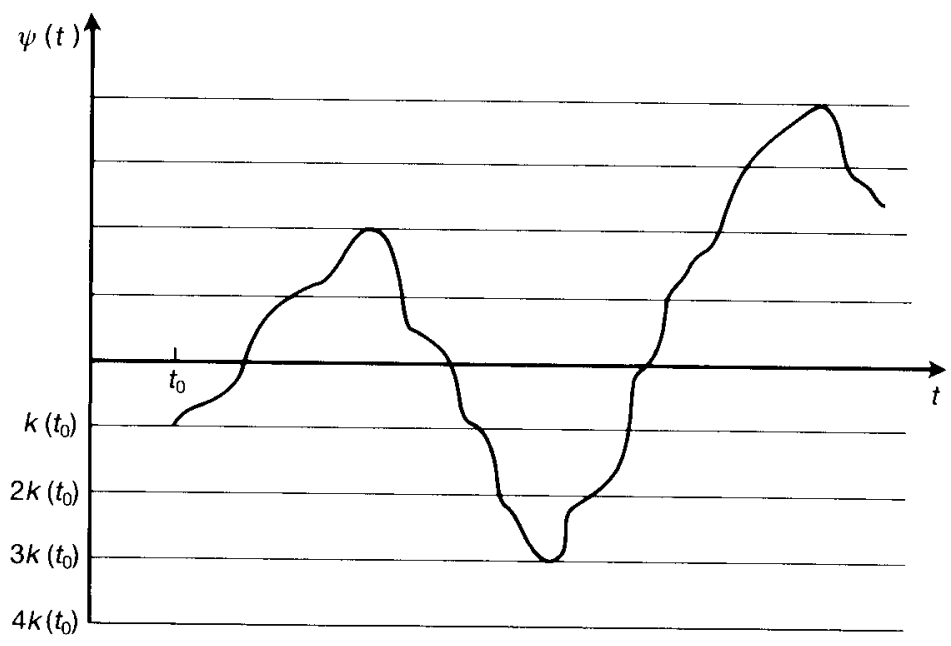

FIG. 1

Note that the consequent switching structure illustrated in Fig. 1 is well-defined since

(i) $\psi(t)$ is monotonic in any interval of $t>t_{0}$ where $S(\cdot)$ is constant,

(ii) $\psi\left(t_{0}\right)=k\left(t_{0}\right)$ and hence the switch times are well defined.

The following lemma gives a basic integral inequality for the output $y(t)=c x(t)$ of the system (3.5), and is used to prove convergence of the switching sequence.

Lemma 3.2 Suppose $(A, b, c) \in \hat{\Sigma}, x_{0} \in \mathbb{R}^{n}$, and $k(\cdot):\left[0, t^{\prime}\right) \rightarrow \mathbb{R}_{+}$is a nondecreasing continuous function, with $0<t^{\prime} \leqslant \infty$. Suppose that on $\left(-\infty, t^{\prime}\right)$ there exists a unique solution $x(\cdot)$ of the closed-loop system

$$
\dot{x}(t)=[A-S(t) k(t) b c] x(t), \quad x\left(t_{0}\right)=x_{0} \quad \text { for } t_{0} \in\left[0, t^{\prime}\right),
$$

where $S(\cdot)$ is defined via the output $y(t)=c x(t)$, the switching decision function $\psi(\cdot)$, and the Algorithm 3.1. Then the following inequality holds true for some suitable $K>0$ and all $t \in\left[t_{0}, t^{\prime}\right)$ :

$$
\begin{aligned}
\frac{1}{2} y(t)^{2} & \leqslant K+K \int_{t_{0}}^{t} y(\tau)^{2} \mathrm{~d} \tau-c b \int_{t_{0}}^{t} S(\tau) k(\tau) y(\tau)^{2} \mathrm{~d} \tau \\
& =K+\int_{t_{0}}^{t} y(\tau)^{2} \mathrm{~d} \tau[K-c b \psi(t)] .
\end{aligned}
$$

Proof. Because of Lemma 2.1, we may assume that (3.7) is of the form (2.5). Then a straightforward calculation yields

$$
\frac{1}{2} \frac{\mathrm{d}}{\mathrm{d} t}\left[y(t)^{2}\right]=A_{1} y(t)^{2}-c b S(t) k(t)^{2}+y(t) A_{2} \eta(t),
$$


and hence

$$
\frac{1}{2}\left[y(t)^{2}-y\left(t_{0}\right)^{2}\right] \leqslant K_{1} \int_{t_{0}}^{t} y(\tau)^{2} \mathrm{~d} \tau-c b \int_{t_{0}}^{t} S(\tau) k(\tau) y(\tau)^{2} \mathrm{~d} \tau+K_{1} \int_{t_{0}}^{t}|y(\tau)|\|\eta(\tau)\| \mathrm{d} \tau
$$

for some suitable $K_{1}>0$. By using (2.7) and applying variations-of-constants to the second equation in (2.5), we obtain for some $K_{2}, \varepsilon>0$ and for $t \geqslant t_{0}$

where

$$
\|\eta(t)\| \leqslant K_{2} \mathrm{e}^{-\varepsilon t}\left\|\eta\left(t_{0}\right)\right\|+L y(\cdot)(t),
$$

$$
L y(\cdot)(t):=\int_{t_{0}}^{t} K_{2} \mathrm{e}^{-\varepsilon(t-s)}|y(s)| \mathrm{d} s .
$$

Since the operator $L$ maps $\mathrm{L}_{2}\left(t_{0}, t^{\prime}\right)$ functions into $\mathrm{L}_{2}\left(t_{0}, t^{\prime}\right)$ functions and is of uniformly bounded norm in $t^{\prime}$ and $t_{0}$, we conclude from (3.9) and (3.10) that

$$
\frac{1}{2} y(t)^{2} \leqslant K_{3}+K_{1} \int_{t_{0}}^{t} y(\tau)^{2} \mathrm{~d} \tau+K_{3}\left(\int_{t_{0}}^{t} y(\tau)^{2} \mathrm{~d} \tau\right)^{\frac{1}{2}}-c b \int_{t_{0}}^{t} S(\tau) k(\tau) y(\tau)^{2} \mathrm{~d} \tau
$$

for some suitable $K_{3}>0$. Since $\alpha \beta \leqslant \alpha^{2}+\beta^{2}$ for $\alpha, \beta \in \mathbb{R}$, (3.11) implies (3.8) and the proof is complete.

REMARK 3.3 (Convergence of the switching mechanism) Under the assumptions of Lemma 3.2, it follows that $\psi(\cdot) \in \mathrm{L}_{\infty}\left(t_{0}, t^{\prime}\right)$. If $\psi(\cdot)$ is unbounded, then the Algorithm 3.1 indicates that

$$
\sup _{t \in\left(t_{0}, t^{\prime}\right)} \psi(t)=\infty \quad \text { and } \inf _{t \in\left(t_{0}, t^{\prime}\right)} \psi(t)=-\infty
$$

This would contradict (3.8). Therefore there exist $\psi_{\infty} \in \mathbb{R}$ and $t_{N}>0$ so that

$$
\lim _{t \rightarrow t^{\prime}} \psi(t)=\psi_{\infty} \quad \text { and } \quad S(t)=S\left(t_{N}\right) \quad \text { for all } t \in\left[t_{N}, t^{\prime}\right) .
$$

To prove the main result of this section, we need a lemma describing the relative growth of $|y(\cdot)|$ and $\|\eta(t)\|$ in the case of $S\left(t_{N}\right) c b<0$ and $k(\cdot)$ unbounded and monotonically nondecreasing.

Lemma 3.4 (Output dominating the state) Suppose $(A, b, c) \in \hat{\Sigma}$ is in the form (2.5) and the assumptions of Lemma 3.2 are satisfied. If $S(t) c b<0$ for all $t \in\left[t_{N}, t^{\prime}\right)$, and $k(\cdot) \notin \mathrm{L}_{\infty}\left(0, t^{\prime}\right)$, then, for every $\varepsilon>0$, there exists some $t_{\varepsilon} \in\left(0, t^{\prime}\right)$ such that

$$
\|\eta(t)\| \leqslant \varepsilon|y(t)| \text { for all } t \in\left[t_{\varepsilon}, t^{\prime}\right) .
$$

Proof. We assume that $x(t) \neq 0$ for all $t \in\left(-\infty, t^{\prime}\right)$; otherwise $x(\cdot) \equiv 0$. Following Owens et al. (1984), we may assume that $b=(1,0, \ldots, 0)^{\top}$ and $c=(c b) b^{\top}$. Put

$$
z(t):=\|x(t)\|^{-1} x(t), \quad V(t):=\left[b^{\top} z(t)\right]^{2} .
$$

It is obvious that $\|z(t)\|=1, b^{\top} z(t)=(c b)^{-1} y(t) /\|x(t)\|$, and $V(t) \in[0,1]$ for all $t \in\left(-\infty, t^{\prime}\right)$. We want to show that, under the stated conditions,

$$
\lim _{t \rightarrow r^{\prime}} V(t)=1 .
$$


If this holds true, then for arbitrary $\bar{\varepsilon}>0$ there exists some $t_{\varepsilon} \in\left(0, t^{\prime}\right)$ such that

$$
1-\bar{\varepsilon} \leqslant(c b)^{-1} \frac{|y(t)|}{\|x(t)\|} \text { for all } t \in\left[t_{\varepsilon}, t^{\prime}\right) .
$$

Using the fact that $\|x\|^{2}=(c b)^{-2} y^{2}+\|\eta\|^{2}$, a straightforward calculation applied to (3.16) yields (3.13).

It remains to prove (3.15). We calculate

$$
\dot{z}=A z-\left(z^{\top} A z\right) z-S k(c b) b^{\top} z\left[b-\left(b^{\top} z\right) z\right],
$$

from which, omitting the argument $t \in\left(t_{N}, t^{\prime}\right)$,

$$
\begin{aligned}
\dot{V} & =\left(2 b^{\top} z\right) b^{\top}\left[A z-\left(z^{\top} A z\right) z-k(c b) b^{\top} z\left(b-b^{\top} z z\right)\right] \\
& =2 b^{\top} z\left[b^{\top} A z-\left(z^{\top} A z\right) b^{\top} z\right]+2 k|c b| V[1-V] .
\end{aligned}
$$

As a consequence, for some $M>0$ independent of $\tau$, we have

$$
\dot{V}(t) \geqslant-M+2 k(t)|c b| V(t)[1-V(t)] \text { for all } t \in\left(t_{N}, t^{\prime}\right) .
$$

Let $I=(a, b) \subset[0,1]$ and $\delta>0$. Since $\lim _{t \rightarrow t^{\prime}} k(t)=\infty$, there exists some $t_{I} \in\left(t_{N}, t^{\prime}\right)$ such that

$$
\dot{V}(t)>\delta \text { for all } t \in\left[t_{l}, t^{\prime}\right) \text { and } V(t) \in I .
$$

This implies

$$
V(t) \in[0, a] \cup[b, 1] \text { for all } t \in\left[\hat{t}, t^{\prime}\right),
$$

where $\hat{t} \in\left[t_{l}, t^{\prime}\right)$ is sufficiently large. Since $I$ was arbitrary, it follows that either $\lim _{t \rightarrow t^{\prime}} V(t)=0$ or $\lim _{t \rightarrow t^{\prime}} V(t)=1$. If the latter holds true, the proof is complete. Thus, it remains to consider the case $\lim _{t \rightarrow l^{\prime}}, V(t)=0$. In this case, for every $\lambda>0$, there exists $t_{\lambda} \in\left(t_{N}, t^{\prime}\right)$ such that

$$
V(t)=(c b)^{-2} \frac{y(t)^{2}}{\|x(t)\|^{2}}<\lambda \quad \text { for all } t \in\left(t_{\lambda}, t^{\prime}\right)
$$

Using $\|x\|^{2}=(c b)^{-2} y^{2}+\|\eta\|^{2}$, it follows that

$$
y(t)^{2}<(c b)^{-2} \frac{\lambda}{1-\lambda}\|\eta(t)\|^{2} \text { for all } t \in\left(t_{\lambda}, t^{\prime}\right) .
$$

Inserting (3.18) into (3.10) yields

$$
\|\eta(t)\| \leqslant K \mathrm{e}^{-\varepsilon t}\left\|\eta\left(t_{\lambda}\right)\right\|+K \lambda \int_{t_{\lambda}}^{t} \mathrm{e}^{-\varepsilon(t-s)}\|\eta(s)\| \mathrm{d} s
$$

for some suitable constant $K>0$. Since $\lambda>0$ can be chosen arbitrarily small, an application of the Bellmann-Gronwall lemma to (3.19) yields an exponentially decaying bound for $\|\eta(\cdot)\|$. But then, by (3.18), $|y(\cdot)|$ is bounded by an exponentially decaying function, which, together with the observation that $\omega(t) \rightarrow 0$ as $t \rightarrow t^{\prime}$, contradicts $k(\cdot) \notin \mathrm{L}_{2}\left(0, t^{\prime}\right)$. This completes the proof of the result. 
Now we are in a position to prove the main result of this section.

Proposition 3.5 Suppose the system $(A, b, c)$ belongs to the class $\hat{\Sigma}$, with $h>0$, and $x_{0} \in \mathbb{R}^{n}$ and $k_{0}>0$ are arbitrary initial data. Then the adaptive mechanism

$$
\begin{aligned}
& k(t)=\max _{0 \leqslant s \leqslant t} \mathrm{e}^{\omega(s) s}\|y(s)\|+k_{0}\left(k_{0} \geqslant 0\right), \\
& \omega(t)=\left\{\begin{array}{cl}
1 & \text { for } t \in[0, h], \\
\frac{1}{1+k(t-h)} & \text { for } t>h,
\end{array}\right. \\
& u(t)=-S(t) k(t) y(t),
\end{aligned}
$$

where $S(\cdot)$ is the switching function defined in the Algorithm 3.1 via the switching decision function (3.6) and the output $y(t)=c x(t)$, produces an exponentially decaying solution of the closed-loop system

$$
\dot{x}(t)=[A-S(t) k(t) b c] x(t), \quad x(0)=x_{0},
$$

with convergent gain

$$
\lim _{t \rightarrow \infty} k(t)=k_{\infty}<\infty
$$

and finally constant switching function

$$
S(t)=\bar{S} \text { for all } t>t_{N} \text { for some } t_{N}>0 .
$$

Moreover, the output satisfies

$$
|y(t)| \leqslant\left(k_{\infty}-k_{0}\right) \mathrm{e}^{-\omega_{\infty} t} \text { for all } t \geqslant 0,
$$

where $\lim _{t \rightarrow \infty} \omega(t)=\omega_{\infty}>0$.

Proof. We proceed in several steps and show the following statements.

(i) If there exists a solution of $(3.21)$ on $\left(-\infty, t^{\prime}\right)$, with $t^{\prime} \leqslant \infty$, then $k(\cdot) \in \mathrm{L}_{\infty}\left(0, t^{\prime}\right)$.

(ii) System (3.21) does not have a finite escape time.

(iii) A solution of (3.21) exists on the whole of $\mathbb{R}$ and is unique.

(iv) There exists $M, \omega>0$ such that the solution $x(\cdot)$ of (3.21) satisfies

$$
\|x(t)\| \leqslant M \mathrm{e}^{-\omega t} \text { for all } t \geqslant 0 \text {. }
$$

If these statements hold true, then (3.24) follows immediately from the construction of the maximum controller and the proposition is proved.

(i) Suppose that there exists a solution $x(\cdot)$ of $(3.21)$ on $\left(-\infty, t^{\prime}\right)$ and that

$$
\lim _{t \rightarrow t^{\prime}} k(t)=\infty \text {, }
$$


noting also that (3.12) holds true. If $S\left(t_{N}\right) c b>0$, then Lemma 2.1 implies the existence of an exponential bound for $\|x(\cdot)\|$. This would contradict (3.22). If $S\left(t_{N}\right) c b<0$, then (3.13) applied to

$$
\frac{1}{2} \frac{\mathrm{d}}{\mathrm{d} t}\left[y(t)^{2}\right]=A_{1} y(t)^{2}-c b S(t) k(t) y(t)^{2}+y(t) A_{2} \eta(t)
$$

(we assume that (3.20) in in the form (2.5)) yields

$$
\frac{1}{2} \frac{\mathrm{d}}{\mathrm{d} t}\left[y(t)^{2}\right] \geqslant\left[A_{1}+\left|c b S\left(t_{N}\right)\right| k(t)-\varepsilon\left\|A_{2}\right\|\right] y(t)^{2} \quad \text { for all } t \in\left[t_{\varepsilon}, t^{\prime}\right),
$$

where $t_{\varepsilon}$ is sufficiently close to $t^{\prime}$. Thus $|y(\cdot)|$ grows exponentially, which contradicts $\psi(\cdot) \in \mathrm{L}_{\infty}\left(0, t^{\prime}\right)$. This proves (i).

(ii) Suppose that for some $t^{\prime}>0$ we have

$$
\limsup _{t \rightarrow t^{\prime}}\|x(t)\|=\infty
$$

Then (i) implies that $k(\cdot) \in \mathrm{L}_{\infty}\left(0, t^{\prime}\right)$. But then the right-hand side of (3.21) would satisfy a global Lipschitz condition on $\left(0, t^{\prime}\right)$, which contradicts (3.26). This proves (ii).

Statements (iii) and (iv) are proved in a similar manner to Proposition 2.2. The proofs are hence omitted for brevity.

\section{Acknowledgement}

To the British Council for travel and subsistence support in pursuing this research.

\section{REFERENCES}

Byrnes, C. I., \& Willems, J. C. 1984 Adaptive stabilization of multivariable linear systems. Proc. 23rd Conf. Decision and Control, Las Vegas, pp. 1574-77.

Hale, J. 1977 Theory of Functional Differential Equations. New York: Springer.

Ilchmann, A., Owens, D. H., \& Prätzel-Wolters, D. 1987 High gain robust adaptive controllers for multivariable systems. Syst. Control Lett. 8, 397-404.

Mårtensson, B. 1986 Adaptive stabilization. Doctoral Dissertation, Lund Institute of Technology, Sweden.

MoRse, A. S. 1983 Recent problems in parameter adaptive control. In: Outils et Modèles Mathèmatiques pour l'Automatique, l'Analyse de Système et le Traitment du Signal (I. D. Landau, ed.), Vol. 3. Paris: Editions du CNRS, pp. 733-40.

Nussbaum, R. D. 1983 Some remarks on a conjecture in parameter adaptive control. Syst. Control Lett. 3, 243-6.

Owens, D. H., Chotai, A., \& Abiri, A. 1984 Parametrization and approximation methods in feedback theory with applications in high-gain, fast-sampling, and cheap-optimal control. IMA J. Math. Control Inf. 1, 147-71.

Owens, D. H., Prätzel-Wolters, D., \& Ilchmann, A. 1987 Positive-real structure and high-gain adaptive stabilization. IMA J. Math. Control Inf. 4, 167-81.

Prätzel-Wolters, D., Owens, D. H., \& Ilchmann, A. 1989 Robust stabilization by high gain feedback and switching. Int. J. Control 49, 1861-68.

Willems, J. C., \& Byrnes, C. 1. 1984 Global adaptive stabilization in the absence of information on the sign of the high frequency gain. In: Lecture Notes in Control and Information Sciences 62, Berlin: Springer, pp. 49-57. 